Published in final edited form as:

Phys Med Biol. 2013 July 7; 58(13): 4455-4469. doi:10.1088/0031-9155/58/13/4455.

\title{
Varying $\mathrm{kVp}$ as a means of reducing CT breast dose to pediatric patients
}

\author{
KB Mathieu ${ }^{1}$, AC Turner ${ }^{2}$, M Khatonabadi $^{2}$, MF McNitt-Gray ${ }^{2}$, CH Cagnon ${ }^{2}$, and DD Cody ${ }^{3}$ \\ DD Cody: dcody@mdanderson.org \\ ${ }^{1}$ Department of Bioengineering, Rice University, Houston, TX 77005 \\ ${ }^{2}$ Department of Biomedical Physics and Department of Radiology, David Geffen School of \\ Medicine, University of California, Los Angeles, Los Angeles, CA 90024 \\ ${ }^{3}$ Department of Imaging Physics, The University of Texas M. D. Anderson Cancer Center, \\ Houston, TX, 77030
}

\begin{abstract}
We investigated the possibility of reducing radiation dose to the breast tissue of pediatric females by using multiple tube voltages within a single $\mathrm{CT}$ examination. The peak kilovoltage $(\mathrm{kVp})$ was adjusted when the x-ray beam was directly exposing the representative breast tissue of a 5-yearold, 10-year-old, and an adult female anthropomorphic phantom; this strategy was called $\mathrm{kVp}$ splitting and was emulated by using a different $\mathrm{kVp}$ over the anterior and posterior tube angles. Dose savings from $\mathrm{kVp}$ splitting were calculated relative to using a fixed $\mathrm{kVp}$ over all tube angles and the results indicated savings in all three phantoms when using $80 \mathrm{kVp}$ over the posterior tube angles regardless of the anterior $\mathrm{kVp}$. Monte Carlo (MC) simulations with and without $\mathrm{kVp}$ splitting were performed to estimate absorbed breast dose in voxelized models constructed from the CT images of pediatric female patients; $80 \mathrm{kVp}$ was used over the posterior tube angles. The MC simulations revealed breast dose savings of between $9.8 \%$ and $33 \%$ from using $\mathrm{kVp}$ splitting compared to simulations using a fixed $\mathrm{kVp}$ protocol with the anterior technique. Before this strategy could be implemented clinically, the development of suitable image reconstruction algorithms and the image quality of scans with $\mathrm{kVp}$ splitting would need further study.
\end{abstract}

\section{Keywords}

computed tomography (PACS code: 87.57.Q); Monte Carlo simulations (PACS code: 87.10.Rt); dosimetry (PACS code: 87.57.uq); pediatric; breast dose

\section{Introduction}

An inverse relationship has been reported between a patient's age at the time of exposure to ionizing radiation and the patient's relative sensitivity to radiation (National Research Council, 1990). Therefore, pediatric patients undergoing computed tomography (CT) scanning are at higher risk of experiencing adverse health effects (e.g., cancer) than adult patients. Additionally, according to the International Commission of Radiological Protection's (ICRP) most recent tissue weighting factors, female breast tissue is among the most radiosensitive tissues (ICRP, 2007). For these reasons, the risk of radiation-induced breast cancer is considered to be especially high for pediatric female patients undergoing CT procedures.

Many user-input scan parameters, including the x-ray tube voltage (in units of $\mathrm{kVp}$ ), current (in units of milliamperes $[\mathrm{mA}]$ ), and exposure time, influence the amount of radiation that patients are exposed to during CT scanning, as well as the quality of the CT images. The $\mathrm{x}-$ 
ray tube current and exposure time are linearly proportional to radiation dose and their adjustment is considered to be the simplest and most predictable means of reducing CT dose (Curry et al., 1990; Kubo et al., 2008). Thus, tube current modulation (TCM) is often employed, which allows for dose reduction according to a patient's anatomy while maintaining consistent image quality throughout the entire examination (Kalender et al., 1999). A form of TCM specifically designed to reduce breast dose by either greatly reducing or completely turning off the x-ray tube current (i.e., $0 \mathrm{~mA}$ ) over the breasts has recently been developed (X-Care; Siemens Healthcare, Forcheim, Germany). Computer simulations have estimated breast dose reductions of nearly 50\% using X-Care (Vollmar and Kalender, 2008); however, due to an increase in the posterior tube current to maintain image quality (in the absence of anterior projection data), higher doses to the spine and bone marrow were also estimated in these simulations.

Because dose is proportional to $\mathrm{kVp}$ to the 2-2.8 power (Curry et al., 1990; Hamberg et al., 2003; Tack et al., 2007)), varying the $\mathrm{kVp}$ during scanning may result in greater dose savings than from adjusting the tube current through TCM or X-Care. However, one drawback of this approach is that the selection of $\mathrm{kVp}$ options is limited to a few values (typically four options), while there are many tube current choices. Furthermore, $\mathrm{kVp}$ affects both beam energy and intensity, and so the relationship between $\mathrm{kVp}$, dose, and image quality is much more complex than the relationship between tube current and dose. Because decreasing $\mathrm{kVp}$ decreases the $\mathrm{x}$-ray beam's intensity, breast dose reduction may be achieved by decreasing $\mathrm{kVp}$. On the other hand, increasing $\mathrm{kVp}$ increases the beam's penetration and breast tissue is relatively superficial, thus breast dose reduction may also be possible by increasing $\mathrm{kVp}$ while proportionally decreasing the current-exposure time product $(\mathrm{mA} \cdot \mathrm{s})$. Although $\mathrm{kVp}$ adjustment typically requires a compensatory adjustment in tube current to achieve consistent image quality, a study by Kim and Newman revealed that diagnostic image quality was maintained when simultaneously reducing both the $\mathrm{kVp}$ and tube current across pediatric protocols (Kim and Newman, 2010).

In this study, we evaluated the possibility of reducing CT breast dose by adjusting $\mathrm{kVp}$ at angular positions of the x-ray tube that would correspond to direct exposure of patients' breast tissue. The $\mathrm{kVp}$ was either raised or lowered when the $\mathrm{x}$-ray tube was over the breasts; both options were tested to fully analyze the complex relationship between surface dose and $\mathrm{kVp}$. Because kVp would effectively "split" into two values during rotations over the breasts (one for anterior angles of incidence and another for posterior angles of incidence) and then converge back to a single $\mathrm{kVp}$ once past the breasts, we referred to this technique as "kVp splitting." Although $\mathrm{kVp}$ splitting involves the use of two kVps within a single scan, this technique is distinct from dual-energy CT, in that data are not acquired for the full rotation at both $\mathrm{kVps}$.

The purpose of this study was to quantify the potential of $\mathrm{kVp}$ splitting for reducing $\mathrm{CT}$ breast dose. Because CT scanners do not currently allow for $\mathrm{kVp}$ splitting, the physical data that could be collected to assess this technique's effectiveness was limited. Thus, Monte Carlo (MC) computer modeling, which has been previously used to estimate glandular breast dose in patient models (Angel et al., 2009), was employed to simulate kVp splitting on voxelized models generated from the CT images of three female pediatric patients of varying size and age. 


\section{Materials and methods}

\subsection{Measured data}

In order to guide the MC simulations, physical data was collected using a 64-slice CT scanner (LightSpeed VCT; GE Healthcare, Waukesha, WI), PMMA cylindrical CT dose index (CTDI) phantoms, and anthropomorphic phantoms.

2.1.1. CTDI-The use of multiple $\mathrm{kVps}$ within a single scan (as in the case of $\mathrm{kVp}$ splitting) can result in discrepancies in image quality. In order to minimize the effect of varying the $\mathrm{kVp}$ and maintain consistent image quality, changes in the $\mathrm{kVp}$ must be accounted for by compensatory changes in other parameters in the scan technique (e.g., the tube current). It is important to determine how these parameters must be adjusted; however, because $\mathrm{CT}$ images cannot be reconstructed from scans with $\mathrm{kVp}$ splitting, image quality could not be directly evaluated in this study. Thus, we instead determined how to maintain dose (in CTDI phantom) when changing the $\mathrm{kVp}$. To do so, exposures were measured at 80 $\mathrm{kVp}, 100 \mathrm{kVp}, 120 \mathrm{kVp}$, and $140 \mathrm{kVp}$ using a $10-\mathrm{cm}$ pencil ionization chamber ( $\mathrm{RadCal}$ Corporation, Monrovia, CA) connected to an electrometer. The ionization chamber was placed at the 12 o'clock peripheral chamber position inside a 16-cm and 32-cm CTDI phantom placed on the patient tabletop; the chamber was positioned at 12:00 because this position most closely represents the location of breast tissue in a patient. For both setups, data was collected in axial scan mode using single 0.5 -s rotations; to match the protocols for scanning pediatric patients at our facility, the small focal spot size, which was controlled by the tube current, the "large body" scan field of view (SFOV), which employed the large bowtie filter, and a $40 \mathrm{~mm}$ beam width were used. The power of $\mathrm{kVp}$ that dose was proportional to was then calculated for each $\mathrm{kVp}$ relative to a clinical $\mathrm{kVp}$ so that subsequent scaling could be done (in 2.1.2 and 2.2.2) when employing $\mathrm{kVp}$ splitting to maintain a constant dose (in CTDI phantom) relative to an original, clinical protocol. To calculate these powers, the fact that exposure is proportional to $\mathrm{kVp}$ to some power (Curry et al., 1990; Hamberg et al., 2003; Tack et al., 2007) was used to set up the following proportion:

$$
\frac{\text { Exposure }_{1}}{\text { Exposure }_{2}}=\left(\frac{\mathrm{kVp_{1 }}}{\mathrm{kVp}_{2}}\right)^{\mathrm{x}} \text {. }
$$

Then, the power of $\mathrm{kVp}, \mathrm{x}$, was calculated using the definition of a logarithm to transform (1) as follows:

$$
\mathrm{x}=\log _{\left(\mathrm{kV \textrm {p } _ { 1 } / \mathrm { kV } _ { 2 } )}\right.}\left(\text { Exposure }_{1} / \text { Exposure }_{2}\right)
$$

where $\left(\mathrm{kVp}_{1} / \mathrm{kVp}_{2}\right)$ is the base of the logarithm. Within the context this study, $\mathrm{kV} \mathrm{p}_{1}$ represented the $\mathrm{kVp}$ used in the original, clinical protocol, which was 100 or $120 \mathrm{kVp}$ for small to medium or large pediatric patients, respectively, and $\mathrm{kVp} 2$ referred to the $\mathrm{kVp}$ used in the $\mathrm{kVp}$ splitting protocol (as later specified in 2.1.2 and 2.2.2); Exposure $1 /$ Exposure $_{2}$ was the ratio of exposures measured for $\mathrm{kVp}_{1}$ and $\mathrm{kVp}_{2}$. Because the 16-cm CTDI phantom was intended to represent small to medium sized pediatric patients, $\mathrm{kVp}_{1}$ for the 16-cm CTDI phantom was $100 \mathrm{kVp}$. Similarly, $\mathrm{kVp}_{1}$ for the $32-\mathrm{cm}$ phantom was $120 \mathrm{kVp}$ to be consistent with our clinical protocol for scanning larger pediatric patients. Table 1 lists the powers of $\mathrm{kVp}$ determined at the 12:00 peripheral chamber position in the CTDI phantoms.

2.1.2. Anthropomorphic phantoms-After the relationships for describing how exposure varied with $\mathrm{kVp}$ were determined (in 2.1.1.), $\mathrm{kVp}$ splitting was evaluated using 
anthropomorphic dosimetry phantoms representative of a 5-year-old, 10-year-old, and an adult upper torso (with breast attachments) (ATOM family; CIRS, Norfolk, VA). Because $\mathrm{kVp}$ splitting is a novel technique that has not been implemented in CT scanners, it was emulated by performing a series of stationary scans over the equivalent of one full rotation and changing the $\mathrm{kVp}$ at angular positions of the $\mathrm{x}$-ray tube that would correspond to direct exposure of patients' breast tissue (as shown in figure 1).

To validate this approach, 18 0.5-s exposures were delivered with the x-ray tube held stationary at evenly-spaced angular positions (in $20^{\circ}$ increments, starting at $0^{\circ}$ ) over the equivalent of a $360^{\circ}$ rotation; each series of stationary scans was collected at $80,100,120$, and $140 \mathrm{kVp}$. Entrance exposures were measured using a Farmer ionization chamber (RadCal Corporation) placed on the surfaces of the anthropomorphic phantoms at the locations representing the sternum. Exposures delivered by each series of stationary scans, which were calculated as the sum of the 18 individual exposure measurements, and the rotating scans (0.5-s axial scans at the same $\mathrm{kVp}$ as the stationary scans) were normalized by exposure time and then compared. Across all three phantoms and all four $\mathrm{kVps}$, the difference in the normalized exposures delivered by the rotating scans and the series of stationary scans ranged from $0.07 \%$ to $1.44 \%$.

Because the exposures measured using the stationary scan method were consistent with those measured for the rotating scans, the stationary scan method was considered to be valid for emulating $\mathrm{kVp}$ splitting in the anthropomorphic phantoms. Thus, the data collected during the validation scans was also used to characterize dose savings from $\mathrm{kVp}$ splitting. To do so, the cumulative exposures from emulated $\mathrm{kVp}$ splitting scans were calculated and then compared to the sum of the exposures (across all 18 stationary scans) for 100 and 120 $\mathrm{kVp}$ for the 5-year-old and 10-year-old phantoms and the adult phantom, respectively; 100 and $120 \mathrm{kVp}$ were used for the fixed $\mathrm{kVp}$ scans because these values were used clinically for scanning small to medium and large pediatric patients, respectively. To calculate exposure for scans with $\mathrm{kVp}$ splitting, the stationary exposures were combined retrospectively for every combination of the four available $\mathrm{kVps}$ over both the anterior and posterior tube angles. While the tube current would typically be adjusted prospectively when changing the $\mathrm{kVp}$ to minimize the impact on image quality or, in our case, dose in CTDI phantom, because a single tube current was used for each phantom across all scans (regardless of $\mathrm{kVp}$ ), the detected exposures were instead scaled retrospectively to account for $\mathrm{kVp}$ splitting. To do so, the cumulative exposures for the anterior (breast) and posterior regions were scaled separately according to the powers of $\mathrm{kVp}$ determined in 2.1.1 by rearranging Eq. (1) as follows:

$$
\text { Exposure }_{1}=\text { Exposure }_{2}\left(\frac{\mathrm{kVp}_{1}}{\mathrm{kVp}_{2}}\right)^{\mathrm{x}} \text {, }
$$

where Exposure Ereferred to the scaled exposure and Exposure $_{2}$ was the cumulative measured exposure for the anterior (exposures detected at tube angles $300^{\circ}, 320^{\circ}, 340^{\circ}, 0^{\circ}$, $20^{\circ}, 40^{\circ}, 60^{\circ}$ [as shown in figure 1]) or posterior region (exposures detected at tube angles between $80^{\circ}$ and $280^{\circ}$ [inclusive]) of the $\mathrm{kVp}$ splitting simulated scan. $\mathrm{kVp}_{1}$ and $\mathrm{kVp} 2$ are defined in the same manner as in Eq. (2), where $\mathrm{kVp}_{1}$ was $100 \mathrm{kVp}$ for the 5-year-old and 10 -year-old phantoms and $120 \mathrm{kVp}$ for the adult phantom and $\mathrm{kVp}_{2}$ was the $\mathrm{kVp}$ used for the anterior or posterior region of the $\mathrm{kVp}$ splitting scan. The power (x) was taken from the values listed in table 1 . The powers determined from data collected in the 16-cm CTDI phantom were used for the anthropomorphic phantoms representing a 5-year-old and a 10year-old (because they were more similar in size to the $16-\mathrm{cm}$ phantom than the $32-\mathrm{cm}$ 
CTDI phantom); likewise, the powers determined from data collected in the 32-cm CTDI phantom were used for the adult phantom.

To determine total exposure from the equivalent of an entire rotation, the scaled exposures calculated for the posterior and anterior regions were summed. Next, the total exposures for the emulated $\mathrm{kVp}$ splitting scans were compared to the cumulative exposures for scans that used a fixed $\mathrm{kVp}\left(\mathrm{kVp}_{1}\right)$ across all 18 stationary scans. Dose savings, which are given in table 2, were determined by calculating the percent difference in exposure between each $\mathrm{kVp}$ splitting scan (for every combination of anterior and posterior $\mathrm{kVps}$ ) and the relevant fixed $\mathrm{kVp}$ scan; a sample calculation is provided in appendix A.

\subsection{Monte Carlo dose estimates}

$\mathrm{MC}$ simulations with and without $\mathrm{kVp}$ splitting were performed to estimate the amount of radiation dose deposited to the glandular breast tissue of three pediatric female voxelized models so that the breast dose-savings potential of $\mathrm{kVp}$ splitting could be evaluated. MC modeling was employed because it provided a more accurate means of estimating breast dose to individual patients and also because it allowed for scans to be simulated that were unable to be physically performed (e.g., helical scanning with $\mathrm{kVp}$ splitting).

2.2.1. Voxelized models-After receiving institution review board approval, three pediatric female patients who had undergone positron emission tomography with CT (PET/ CT) (Discovery STE; GE Healthcare) and were considered to be representative of a small, medium, and large pediatric female were identified and used to construct voxelized patientbased models for MC modeling. PET/CT rather than diagnostic CT images were used because a single, continuous set of images without intravenous or oral contrast was acquired. Although the PET/CT images had a larger voxel size than is typical of diagnostic CT images, because the voxelized models generated from the images were somewhat coarse, the large voxel size was not considered to be an issue. The patients chosen to represent the small, medium, and large pediatric patients were 3 years old (height: $102 \mathrm{~cm}$; weight: 15 $\mathrm{kg}$ ), 10 years old (height: $144 \mathrm{~cm}$; weight: $37 \mathrm{~kg}$ ), and 17 years old (height: $175 \mathrm{~cm}$; weight: $72 \mathrm{~kg}$ ), respectively, at the time of scanning. Both the CT image data and the raw CT data were collected for these three patients, and, from the raw data, the specific TCM schemes used on each patient were extracted. Techniques used to scan the patients are listed in table 3.

Voxelized models of the three patients were developed by identifying the voxels in the patients' CT images that contained glandular breast or lung tissue; a pediatric radiologist was consulted to ensure proper identification of the patients' breast tissue. Lung tissue was also contoured because it is among the most radiosensitive organs (according to the ICRP tissue weighting factors; ICRP, 2007) and it is in the same scan plane as breast tissue. Figure 2 shows contouring of both the lung and breast tissue in one CT image for each of the three patients. The PET/CT examinations were performed with the patients' arms positioned next to the trunk (as seen in Figure 2); thus, the patients' arms were present in the voxelized models.

2.2.2. Simulations-An MC computer model that uses the MC N-Particle code eXtended version 2.6d (MCNPX) (Turner et al., 2009; Waters, 2003) was employed to estimate the amount of radiation dose deposited to the breast tissue for a given voxelized patient model (generated from the CT data of the small, medium, or large pediatric patient). Breast dose has been previously quantified in patient-based models (GSF; Neuherberg, Germany) using the MCNPX model (DeMarco et al., 2007). However, because kVp splitting has not been previously simulated, it was first validated. To perform this validation, two separate 
simulations (one over the anterior and a second over the posterior region) were performed as in the phantom scans. The dose estimates obtained from these two simulations were then combined to estimate total absolute dose (in milligray [mGy]) for a full scan using the following equation:

$$
\text { Dose }_{\text {total }}=\left(\text { Dose }_{\text {anterior }}+\text { Dose }_{\text {posterior }}\right) \cdot \# \text { of rotations, }
$$

where and can each be estimated as follows:

$$
\text { Dose }=\mathrm{MC} \text { Output } \cdot \mathrm{NF} \cdot \mathrm{mAs} \cdot \frac{\text { Angular coverage }}{360^{\circ}}
$$

where $\mathrm{MC}$ output is the output of the $\mathrm{MC}$ simulation for the anterior or posterior region, $\mathrm{NF}$ is the normalization factor, which is dependent on the $\mathrm{kVp}$, collimation, and scanner model and converts output of the MC simulation to dose in mGy (as described by (DeMarco et al., 2005), mAs is the tube current-exposure time product used across the anterior or posterior region of the simulation, and angular coverage is the coverage (in degrees) of the anterior or posterior region. The estimated dose obtained through $\mathrm{MC}$ modeling of $\mathrm{kVp}$ splitting in a 32-cm CTDI phantom using $120 \mathrm{kVp}$ for posterior angles and $80 \mathrm{kVp}$ for anterior angles was validated within $6.1 \%$ of physical data (not shown); validation was done using a CTDI rather than an anthropomorphic phantom based on prior validation work.

Rather than performing time-consuming MC simulations of $\mathrm{kVp}$ splitting for all $\mathrm{kVp}$ combinations, results from the anthropomorphic phantoms were used to dictate the optimal anterior and posterior $\mathrm{kVps}$ to be used in the simulations. The 5-year-old, 10-year-old, and adult anthropomorphic phantoms were matched with the small, medium, and large pediatric patients, respectively, when determining which $\mathrm{kVps}$ would be used in the simulations for each patient. Based on the results given in table 2, the $\mathrm{kVp}$ used in the original, clinical protocol (i.e., $100 \mathrm{kVp}$ for the small and medium patient models and $120 \mathrm{kVp}$ for the large patient model) was used over the anterior tube angles and the lowest $\mathrm{kVp}$ available (i.e., 80 $\mathrm{kVp}$ ) was used over the posterior tube angles for all three patient models. This combination of $\mathrm{kVps}$ produced the greatest dose savings in the pediatric phantoms and the second largest dose savings in the adult phantom. The posterior and anterior regions were defined according to which x-ray tube angles (using the fan beam's central ray) directly exposed the breast tissue with the patient positioned "as is" within the 50-cm display field of view. Thus, although the angular coverage for off-center patients was different than if the patient had been centered, the simulations were performed without adjusting the patients' position within the field of view. For the small patient, the angular coverage of breast tissue was $230^{\circ}$ when centered "as is" within the 50-cm display field of view; however, if the patient had been properly centered, her breast tissue would have only covered a $130^{\circ}$ angular region.

The clinical protocols (listed in table 3 ) were followed as closely as possible when performing the simulations; however, several factors were changed, including the scanner type, the scan extent, the x-ray tube start angle, and the use of TCM. Although a Discovery STE PET/CT scanner was used to collect the patient data, a GE LightSpeed VCT was used in the simulations because an equivalent-source MC model has not yet been coded for the GE 16-slice scanner used in the Discovery STE PET/CT scanner. To reduce simulation times, rather than simulating the entire scan extent, the scan extent used in the simulations was based on the z-axis locations where breast tissue could be visualized in the CT images plus one beam width (i.e., $40 \mathrm{~mm}$ ) before and after the region containing breast tissue (see figure 3); the additional beam widths were included to ensure complete coverage of the breast tissue. The angular position of the $\mathrm{x}$-ray tube was set to equal $0^{\circ}$ (i.e., the top of the 
gantry) at the z-axis location which corresponded to the center of the simulated scan extent; the start angle was then calculated based on the beam width, pitch, and the distance (along the z-axis) between the start and center of the simulated scan length. TCM was not simulated as this would have added an additional layer of difficulty and was unnecessary for demonstrating the potential of $\mathrm{kVp}$ splitting. Instead, the actual patient TCM schemes (taken from the raw CT data) were used to calculate the average tube currents over the simulated scan extent (as seen in figure 4) and this value was used in Eq. (5) when calculating dose from the output of the MC simulations. For the small, medium, and large patients, the average currents over the scan extent simulated (i.e., images showing breast tissue with a border of one beam width) were calculated to be $65 \mathrm{~mA}, 118 \mathrm{~mA}$, and $108 \mathrm{~mA}$, respectively. Table 4 lists the technical factors and other information relevant to the simulations.

After determining dose for the $\mathrm{kVp}$ splitting simulations, breast and lung dose estimates were also obtained for a fixed $\mathrm{kVp}$ protocol (using the anterior techniques listed in table 4). To quantify the dose-savings potential of $\mathrm{kVp}$ splitting, we compared dose (through percent difference) for a full simulation using the fixed $\mathrm{kVp}$ (and tube current) protocol to the dose for simulations with $\mathrm{kVp}$ splitting. A sample calculation describing the full process of calculating dose from the output of the MC simulation to calculating the percent difference is provided in appendix $\mathrm{B}$.

\section{Results}

Results of the MC simulations for all three patient models appear in table 5. Lung and breast dose estimates are reported for simulations with and without $\mathrm{kVp}$ splitting along with the estimated percentage of dose savings from using $\mathrm{kVp}$ splitting. Scans simulated with $\mathrm{kVp}$ splitting had breast dose savings that ranged from $9.8 \%$ to $33 \%$, or $0.41-0.94 \mathrm{mGy}$, compared with scans simulated using a fixed $\mathrm{kVp}$. Lung dose was generally not affected by $\mathrm{kVp}$ splitting; however, while the entire lung was contoured in the voxelized patient models and included in the dose estimate, the scan extent simulated did not necessarily directly expose all of the patients' lung tissue. The estimated statistical error in the dose estimates obtained through the MC simulations ranged from 0.03 to $0.26 \%$.

\section{Discussion}

$\mathrm{kVp}$ splitting was estimated in this study to lower CT breast dose in three pediatric females by between $9.8 \%$ and $33 \%$ while presumably maintaining constant image noise. While TCM allows for dose reduction according to estimated tissue density and x-ray path length through the patient, $\mathrm{kVp}$ splitting allows for the breast to be specifically targeted for dose reduction, which is important because it is susceptible to cancer.

This study had several limitations, including the absence of image quality analysis. While we attempted to control the effect of $\mathrm{kVp}$ splitting on image noise (by scaling the tube current used in the $\mathrm{MC}$ simulations with the $\mathrm{kVp}$ ), we were unable to directly evaluate image quality. Thus, we cannot be certain that the powers we used for the scaling would maintain image noise and because the tube current settings for irradiation from the posterior angles were determined from these powers, it is possible that greater or lesser dose savings could actually be achieved while maintaining consistent image quality. If we had not scaled the posterior tube currents based on the powers of $\mathrm{kVp}$ we determined and had used the anterior tube current for both $\mathrm{kVps}$, dose to the breast and lung in the three voxelized models would have been reduced by $42-60 \%$ and $15-26 \%$, respectively. Not increasing the current to compensate for reducing the $\mathrm{kVp}$ may be reasonable for pediatric patients because the compensatory tube current increase is thought to be less important for maintaining image quality in smaller patients (Siegel et al., 2004). Another study limitation was the differences 
across the anthropomorphic phantom scans and the MC simulations, including the dose measurement location, improper patient centering, and the tube angles over which each $\mathrm{kVp}$ was used. Although this could explain the discrepancies in dose savings between the phantom measurements and the patient simulations (i.e., $5.3 \%$ dose savings from $\mathrm{kVp}$ splitting in the 5-year-old anthropomorphic phantom versus $33 \%$ breast dose savings in the small voxelized patient model for the same anterior and posterior $\mathrm{kVps}$ ), the observed discrepancies can also be attributed to the fact that anthropomorphic phantoms do not fully represent human anatomy. Since the $\mathrm{kVps}$ used in the MC simulations were chosen based on the anthropomorphic phantom results and the percentages of dose savings observed in the phantoms were inconsistent with those observed in the simulations, we cannot be sure that the optimal combinations of $\mathrm{kVps}$ were used in the simulations. Thus, while the least dose savings was observed for the medium-sized patient model, because this patient was larger than the 10-year-old anthropomorphic phantom and the $16 \mathrm{~cm}$ CTDI phantom, matching to the $\mathrm{kVps}$ used on the larger phantoms may have been more appropriate. Moreover, only three patient models were evaluated in this study and the breast dose savings across these patients was varied. In light of these inconsistencies and because the physical data that can be collected to evaluate $\mathrm{kVp}$ splitting is limited, further MC analysis across a greater sample of pediatric patients is needed in the future. Additionally, while this study related to pediatric patients, because the large pediatric patient model was adult-sized, evaluation of the breast dose-saving potential of $\mathrm{kVp}$ splitting in adults should also be investigated. Such future investigations should also remove the patients' arms from the voxelized models because patients typically have their arms raised above their heads during routine CT examinations. Presence of the patients' arms may have influenced the absolute dose estimates obtained in this study (due to attenuation and scatter). In order to obtain more accurate dose estimates, the arms of the voxelized patient models, which were positioned next to the trunk, could be contoured and identified as air in the future. Because all simulations performed in this study included the patients' arms, we do not believe this had a large impact on our results in terms of the relative dose savings or the dose savings as a percentage. Furthermore, we do not believe that inclusion of the arms affected our proof of concept that $\mathrm{kVp}$ splitting may permit breast dose reduction.

To the best of our knowledge, dose reduction has not been previously quantified for $\mathrm{kVp}$ splitting; however, the use of tube voltage modulation has been suggested (Goldman, 2007). Furthermore, at least one study has addressed the issue of image reconstruction in scans acquired with tube voltage modulation (Ritschl et al., 2010). By applying an empirical cupping correction algorithm to the raw CT data, Ritschl et al. showed that equivalent image quality could be achieved in images acquired with tube voltage modulation compared to images acquired using a fixed $\mathrm{kVp}$. While image quality was not evaluated in our study, image reconstruction would likely pose the greatest challenge to implementation of $\mathrm{kVp}$ splitting. Besides applying the empirical cupping correction algorithm, another potential image reconstruction solution would be to use a single $\mathrm{kVp}$ within each rotation (i.e., to flip from one $\mathrm{kVp}$ to another rather than to split the $\mathrm{kVp}$ into two different values); this approach would be similar to z-axis TCM. However, the dose savings we obtained in anthropomorphic phantoms were inconsistent when flipping to a different $\mathrm{kVp}$ for the entire rotation exposing the breasts (e.g., dose savings was achieved using $80 \mathrm{kVp}$ for the entire rotation in the pediatric anthropomorphic phantoms, but not in the adult phantom; data not shown). Furthermore, while flipping the $\mathrm{kVp}$ would resolve issues with image reconstruction, the CT numbers would vary between images, which could lead to problems with interpretation of these values over an entire exam. If the issues with reconstruction and $\mathrm{CT}$ number correction were resolved, $\mathrm{kVp}$ splitting with more than two $\mathrm{kVps}$ could also be explored. Additionally, while only four $\mathrm{kVp}$ options are currently available on most scanners, in the future, additional $\mathrm{kVp}$ options (e.g., $60 \mathrm{kVp}$ ) could potentially be added to expand the selection of $\mathrm{kVp}$ splitting schemes. Finally, if $\mathrm{kVp}$ splitting were implemented in 
CT scanners, a means of identifying the breast tissue or other sensitive organs would be needed. One means of identifying target tissues would be for technologists to locate and mark specific anatomical landmarks, possibly from the patient's localizer image. Then, appropriate age- and/or size-based presets could be selected which specify the anterior and posterior techniques and $\mathrm{kVp}$ splitting could be implemented over an angular region matching the specific anatomical location of each patient's breast tissue.

In the future, the voxelized patient models used in this study could be expanded in to include other superficial radiosensitive organs, like the lens of the eye and the thyroid. Furthermore, organs within the same scan plane as the breast (e.g., the spine) could also be contoured. Although spine dose was not considered in this study, it should be evaluated in the future to ensure that $\mathrm{kVp}$ splitting does not increase spine dose (as X-Care has been shown to do (Vollmar and Kalender, 2008)).

\section{Conclusion}

Breast tissue is one of the most radiosensitive tissues in the human body. The results of this study indicate that CT breast dose could be reduced by as much as $33 \%$ in pediatric patients by varying the tube voltage during those rotations that directly expose breast tissue. The development of novel techniques like $\mathrm{kVp}$ splitting for reducing pediatric breast dose may help to prevent radiation-induced breast cancer in adult women who undergo CT scans during childhood.

\section{Acknowledgments}

This research was supported by NIH grants CA016672 and R01-EB0048989. The authors wish to acknowledge Dr. Adam Chandler for his assistance with extracting the TCM schemes from the raw CT data files and Dr. Nancy Fitzgerald for her assistance with identifying the breast tissue in the CT images.

\section{Appendix A}

\section{Sample calculation for determining dose savings for scans of the anthropormorphic phantoms with $\mathrm{kVp}$ splitting}

To determine the total exposure for a $\mathrm{kVp}$ splitting emulated scan using $120 \mathrm{kVp}$ over the anterior (breast) region and $80 \mathrm{kVp}$ over the posterior region on the 5-year-old anthropomorphic phantom, we first calculated the individual scaled exposures for the anterior and posterior regions relative to the $\mathrm{kVp}$ used in the original, clinical protocol (i.e., $100 \mathrm{kVp}$ ) using Eq. (3) and the appropriate power (x) listed in table 1:

$$
\text { Exposure }_{1, \text { anterior }}=\text { Exposure }_{2}\left(\frac{100}{120}\right)^{2.284},
$$

where Exposure $_{2}=12121 \mathrm{mR}$ and represents the sum of the seven exposures detected at tube angles $300^{\circ}, 320^{\circ}, 340^{\circ}, 0^{\circ}, 20^{\circ}, 40^{\circ}$, and $60^{\circ}$. Thus, Exposure ${ }_{1 \text {, anterior }}=7993 \mathrm{mR}$.

Repeating this calculation for the posterior region with the appropriate values:

$$
\text { Exposure }_{1, \text { posterior }}=1373 \mathrm{mR} \cdot\left(\frac{100}{80}\right)^{2.566}=2434 \mathrm{mR} \text {. }
$$


To calculate the total exposure, the scaled exposures for the anterior and posterior regions were summed:

Exposure $_{\text {total }}=$ Exposure $_{1, \text { anterior }}+$ Exposure $_{1, \text { posterior }}=7993 \mathrm{mR}+2434 \mathrm{mR}=10427 \mathrm{mR}$.

Next, we compared the exposure for the $\mathrm{kVp}$ splitting emulated scan to the exposure for the fixed $\mathrm{kVp}$ clinical protocol, which was the sum of the exposures detected at all 18 tube angles for $100 \mathrm{kVp}$ (i.e., $10837 \mathrm{mR}$ ), through a percent difference calculation:

$$
\text { Percent difference }=\frac{10427 \mathrm{mR}-10837 \mathrm{mR}}{10837 \mathrm{mR}} \cdot 100 \%=-3.8 \% \text {. }
$$

\section{Appendix B}

\section{Sample calculation for estimating dose savings from a Monte Carlo simulation of $\mathbf{k V p}$ splitting}

To calculate the breast dose for a Monte Carlo simulated scan with $\mathrm{kVp}$ splitting using 100 $\mathrm{kVp}$ over the anterior (breast) region (from $245-115^{\circ}$, clockwise) and $80 \mathrm{kVp}$ over the posterior region (from $115-245^{\circ}$, clockwise) with the small patient model, we must first convert the output of the MC simulation to absolute dose. Because this is done separately for each of the two regions with $\mathrm{kVp}$ splitting, we first calculate the absolute breast dose for the anterior (breast) region of the simulation using Eq. (5) and the appropriate values taken from table 4:

$$
\text { Dose }_{\text {anterior }}=\left(1.101 \cdot 10^{-13}\right) \cdot \frac{1.046 \cdot 10^{11} \mathrm{mGy}}{\mathrm{mAs}} \cdot(65.4 \mathrm{~mA} \cdot 0.5 \mathrm{~s}) \cdot \frac{230^{\circ}}{360^{\circ}}=0.24 \mathrm{mGy} \text {. }
$$

Repeating this calculation for the posterior region:

$$
\text { Dose }_{\text {posterior }}=\left(6.463 \cdot 10^{-13}\right) \cdot \frac{5.61 \cdot 10^{10} \mathrm{mGy}}{\mathrm{mAs}} \cdot(116 \mathrm{~mA} \cdot 0.5 \mathrm{~s}) \cdot \frac{130^{\circ}}{360^{\circ}}=0.76 \mathrm{mGy} \text {. }
$$

To calculate the total exposure for an entire rotation, the absolute doses for the anterior and posterior regions are combined according to Eq. (4):

$$
\text { Dose }_{\text {total }}=(0.24 \mathrm{mGy}+0.76 \mathrm{mGy}) \cdot \text { \#of rotations. }
$$

Scan extent (rather than number of rotations) is listed in table 4 and we can calculate the number of rotations from the scan extent according to the following equation:

$$
\text { \#of rotations }=\frac{\text { scan extent }}{\text { pitch } \cdot \text { beam width }}
$$

Therefore, 


$$
\text { \# of rotations }=\frac{104.6 \mathrm{~mm}}{1.375 \cdot 40 \mathrm{~mm}}=1.90 \text { rotations. }
$$

And so,

$$
\text { Dose }_{\text {total }}=(1.00 \mathrm{mGy}) \cdot 1.90 \text { rotations }=1.90 \mathrm{mGy} \cdot \quad \text { (A.1) }
$$

In order to calculate percent difference between the $\mathrm{kVp}$ splitting simulated scan and the simulated scan using the fixed $\mathrm{kVp}$ protocol, we must first calculate dose for the fixed $\mathrm{kVp}$ protocol using a combination of Eq.s (4) and (5), where:

$$
\text { Dose }=\mathrm{MC} \text { Output } \cdot \mathrm{NF} \cdot \mathrm{mAs} \cdot \# \text { of rotations. (A.2) }
$$

Thus, absolute breast dose for a full scan at $100 \mathrm{kVp}$ is:

$$
\text { Dose }=\left(4.371 \cdot 10^{-13}\right) \cdot \frac{1.046 \cdot 10^{11} \mathrm{mGy}}{\mathrm{mAs}} \cdot(65.4 \mathrm{~mA} \cdot 0.5 \mathrm{~s}) \cdot 1.90 \text { rotations }=2.85 \mathrm{mGy} \text {. }
$$

Finally, the percent difference is calculated as:

$$
\text { Percent difference }=\frac{1.90 \mathrm{mGy}-2.85 \mathrm{mGy}}{2.85 \mathrm{mGy}} \cdot 100 \%=-33 \% \text {. }
$$

\section{References}

Angel E, Yaghmai N, Jude CM, Demarco JJ, Cagnon CH, Goldin JG, Primak AN, Stevens DM, Cody $\mathrm{DD}$, McCollough CH, McNitt-Gray MF. Monte Carlo simulations to assess the effects of tube current modulation on breast dose for multidetector CT. Phys Med Biol. 2009; 54:497-512. [PubMed: 19124953]

Curry, TS.; Dowdey, JE.; Murry, RC.; Christensen, EE. Christensen's physics of diagnostic radiology. Philadelphia: Lea \& Febiger; 1990.

DeMarco JJ, Cagnon CH, Cody DD, Stevens DM, McCollough CH, O'Daniel J, McNitt-Gray MF. A Monte Carlo based method to estimate radiation dose from multidetector CT (MDCT): cylindrical and anthropomorphic phantoms. Phys Med Biol. 2005; 50:3989-4004. [PubMed: 16177525]

DeMarco JJ, Cagnon CH, Cody DD, Stevens DM, McCollough CH, Zankl M, Angel E, McNitt-Gray MF. Estimating radiation doses from multidetector CT using Monte Carlo simulations: effects of different size voxelized patient models on magnitudes of organ and effective dose. Phys Med Biol. 2007; 52:2583-97. [PubMed: 17440254]

Goldman LW. Principles of CT: radiation dose and image quality. J Nucl Med Technol. 2007; 35:21325. quiz 26-8. [PubMed: 18006597]

Hamberg LM, Rhea JT, Hunter GJ, Thrall JH. Multi-detector row CT: radiation dose characteristics. Radiology. 2003; 226:762-72. [PubMed: 12616020]

ICRP. The 2007 Recommendations of the International Commission on Radiological Protection. ICRP publication 103. Ann ICRP. 2007; 37:1-332.

Kalender WA, Wolf H, Suess C, Gies M, Greess H, Bautz WA. Dose reduction in CT by online tube current control: principles and validation on phantoms and cadavers. Eur Radiol. 1999; 9:323-28. [PubMed: 10101657] 
Kim JE, Newman B. Evaluation of a radiation dose reduction strategy for pediatric chest CT. AJR Am J Roentgenol. 2010; 194:1188-93. [PubMed: 20410401]

Kubo T, Lin PJ, Stiller W, Takahashi M, Kauczor HU, Ohno Y, Hatabu H. Radiation dose reduction in chest CT: a review. AJR Am J Roentgenol. 2008; 190:335-43. [PubMed: 18212218]

National Research Council. Health effects of exposure to low levels of ionizing radiation (BEIR V). Washington, DC: 1990.

Ritschl L, Bergner F, Fleischmann C, Kachelriess M. Water calibration for CT scanners with tube voltage modulation. Phys Med Biol. 2010; 55:4107-17. [PubMed: 20601772]

Siegel MJ, Schmidt B, Bradley D, Suess C, Hildebolt C. Radiation dose and image quality in pediatric CT: effect of technical factors and phantom size and shape. Radiology. 2004; 233:515-22. [PubMed: 15358847]

Tack, D.; Gevenois, PA. SpringerLink (Online service). Radiation Dose from Adult and Pediatric Multidetector Computed Tomography. Berlin, Heidelberg: Springer-Verlag Berlin Heidelberg; 2007.

Turner AC, Di Z, Kim HJ, DeMarco JJ, Cagnon CH, Angel E, Cody DD, Stevens DM, Primak AN, McCollough CH, McNitt-Gray MF. A method to generate equivalent energy spectra and filtration models based on measurement for multidetector CT Monte Carlo dosimetry simulations. Med Phys. 2009; 36:2154-64. [PubMed: 19610304]

Vollmar SV, Kalender WA. Reduction of dose to the female breast in thoracic CT: a comparison of standard-protocol, bismuth-shielded, partial and tube-current-modulated CT examinations. Eur Radiol. 2008; 18:1674-82. [PubMed: 18414873]

Waters L. Los Alamos National Laboratory Report No LA-UR-03-2202. 2003 


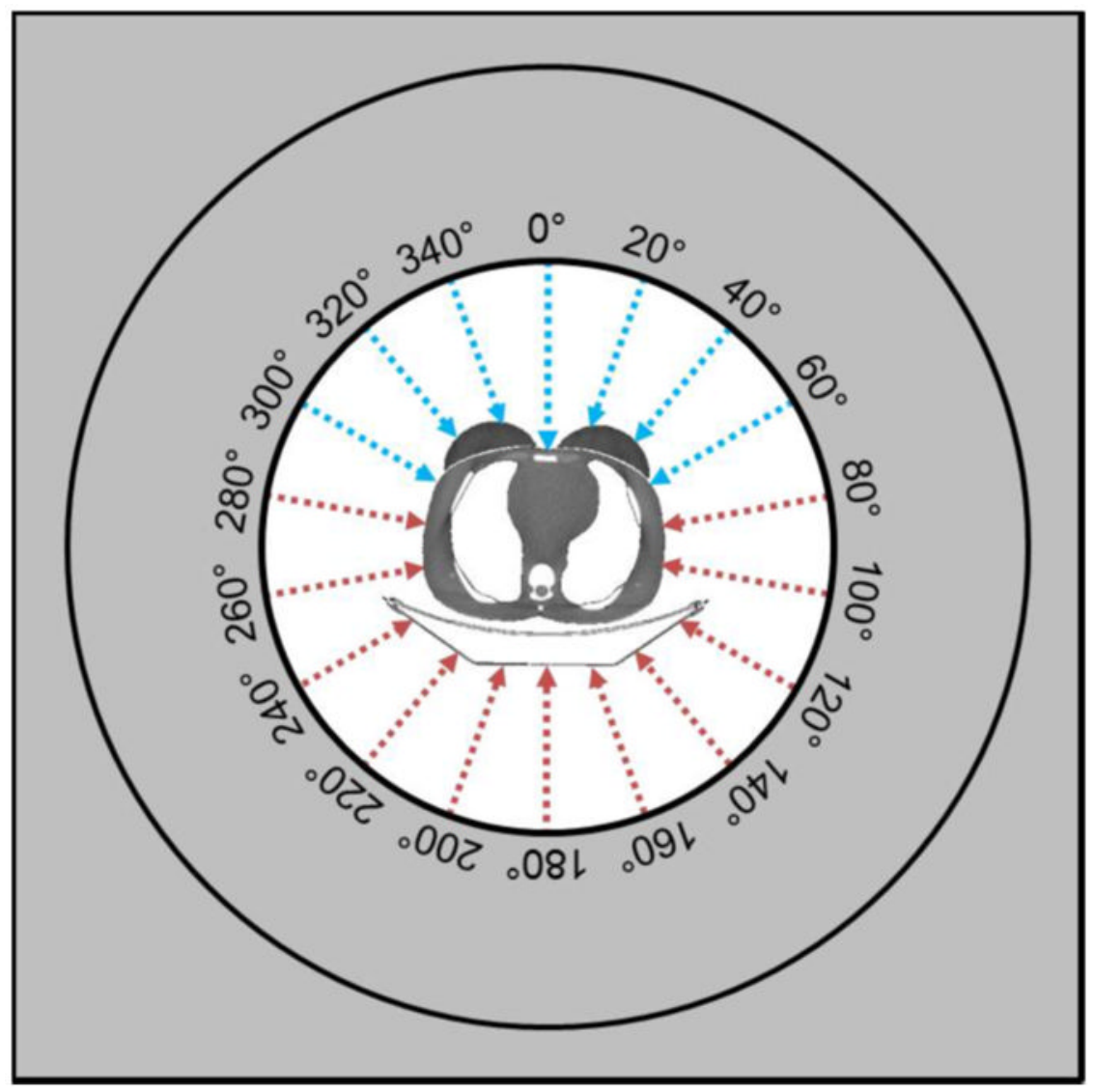

Figure 1.

A series of 18 stationary scans were performed in $20^{\circ}$ angular increments from $0^{\circ}$ to $340^{\circ}$ to emulate a $360^{\circ}$ rotation with $\mathrm{kVp}$ splitting. The central ray of the $\mathrm{x}$-ray fan beam at each angular increment is shown as a red or blue arrow; the blue arrows represent scans at the anterior tube angles while the red arrows represent scans at the posterior tube angles. 

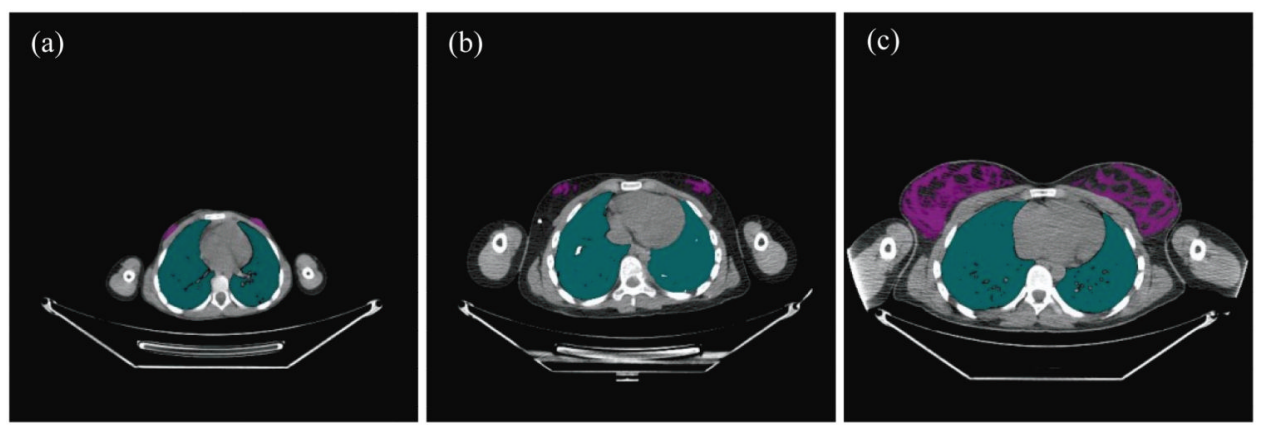

Figure 2.

Lung (teal) and glandular breast tissue (purple) were contoured for the (a) small, (b) medium, and (c) large pediatric patients. All of the images are shown with a 50-cm display field of view to allow for direct comparison and visualization of the differences in the patient sizes. The patient diameters (measured from left-to-right) are (a) 18, (b) 26.5, and (c) $31 \mathrm{~cm}$, which can be compared to diameters of $17.5,21$, and $32.5 \mathrm{~cm}$ for the 5-year-old, 10year-old, and adult anthropomorphic phantoms. 

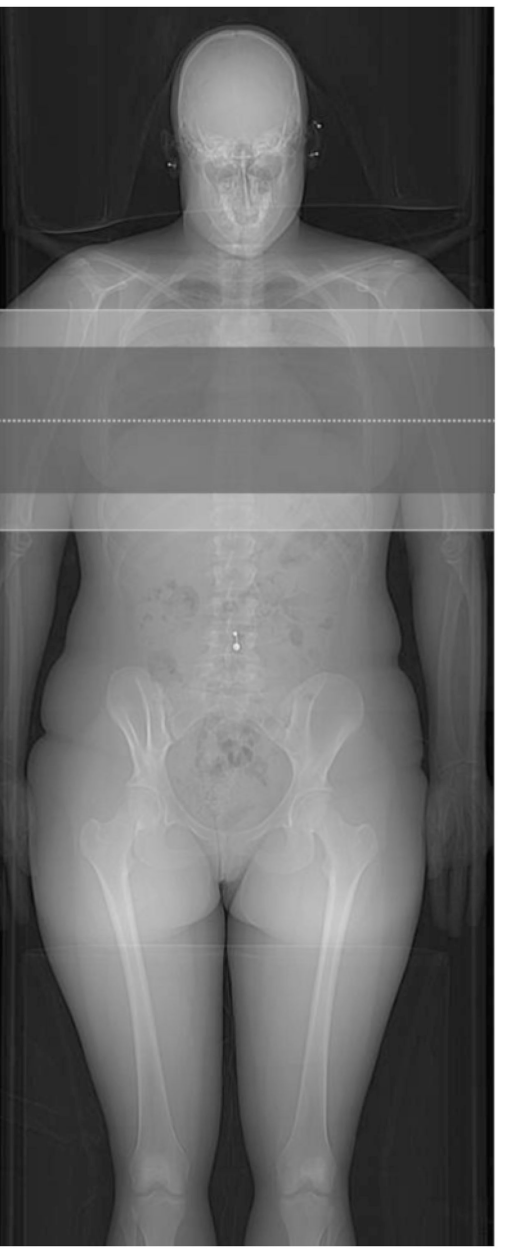

Figure 3.

Highlighting shows the $\mathrm{z}$-axis coverage of the breast tissue (darker highlighted region) and the scan extent used in the simulations (lighter highlighted region) for the large pediatric patient. The dashed line in the middle of the scan extent is the $\mathrm{z}$-axis position at which the $\mathrm{x}$ ray tube angle was set to $0^{\circ}$. 


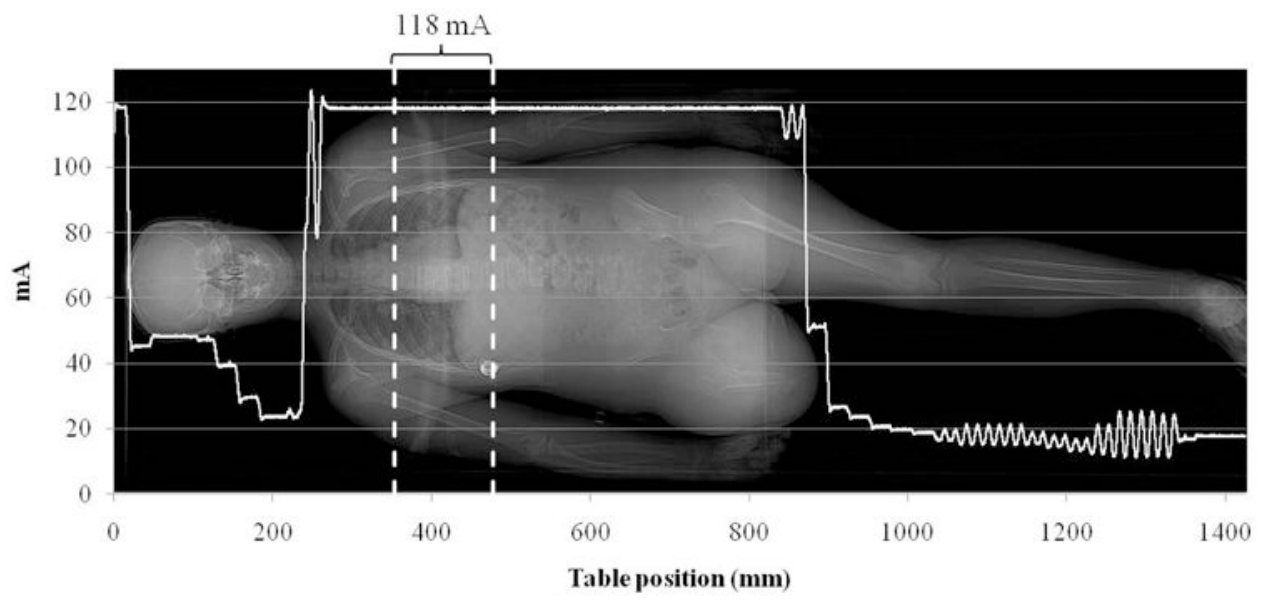

Figure 4.

The TCM scheme (obtained from the raw CT data) used to scan the medium-sized pediatric patient superimposed on the patient's localizer image. Because the Monte Carlo simulation of $\mathrm{kVp}$ splitting was performed over the breast region only (enclosed between the dashed lines), the average tube current over this region was used when converting the output of the simulation to dose; for this patient, the average current was $118 \mathrm{~mA}$. 


\section{Table 1}

Powers of $\mathrm{kVp}$ were calculated using Eq. (2) from the exposures measured at the 12:00 chamber position in a $16-\mathrm{cm}$ or 32-cm CTDI phantom; $\mathrm{kVp} 1$ values of 100 and $120 \mathrm{kVp}$ were used for the 16- and 32-cm CTDI phantoms, respectively.

\begin{tabular}{lll}
\hline $\mathbf{k V p _ { 1 }} / \mathbf{k V p _ { 2 }}$ & 16-cm phantom & 32-cm phantom \\
\hline $100 / 80$ & 2.57 & - \\
$100 / 120$ & 2.28 & - \\
$100 / 140$ & 2.25 & - \\
$120 / 80$ & - & 2.66 \\
$120 / 100$ & - & 2.46 \\
$120 / 140$ & - & 2.22 \\
\hline
\end{tabular}




\section{Table 2}

Percentage of dose savings from using $\mathrm{kVp}$ splitting in anthropomorphic phantoms representing a 5-year-old, 10-year-old, and adult relative to a scan at the original, clinical $\mathrm{kVp}\left(\mathrm{kVp}_{1}\right)$; negative values (bold-faced) indicate dose savings. Exposures were scaled across $\mathrm{kVps}$ using Eq. (3) and the powers listed in table 1, which were calculated from exposures measured at 12:00 in a 16-cm CTDI phantom (for the pediatric phantoms) or 32-cm CTDI phantom (for the adult phantom).

\begin{tabular}{|c|c|c|c|}
\hline $\mathrm{kVp}_{1}{ }^{a} / \mathbf{k V p _ { 2 } \text { (anterior } \& \text { posterior) }}$ & 5-year-old & 10-year-old & Adult \\
\hline 100 or $120 / 80 \& 100$ & $-0.2 \%$ & $-0.2 \%$ & $4.1 \%$ \\
\hline 100 or $120 / 80 \& 120$ & $1.7 \%$ & $1.7 \%$ & $5.6 \%$ \\
\hline 100 or $120 / 80 \& 140$ & $2.8 \%$ & $2.7 \%$ & $6.5 \%$ \\
\hline 100 or $120 / 100 \& 80$ & $-5.3 \%$ & $-4.5 \%$ & $-3.3 \%$ \\
\hline 100 or $120 / 100 \& 120$ & $1.9 \%$ & $1.9 \%$ & $1.7 \%$ \\
\hline 100 or $120 / 100 \& 140$ & $3.0 \%$ & $2.9 \%$ & $2.7 \%$ \\
\hline 100 or $120 / 120 \& 80$ & $-3.8 \%$ & $-3.1 \%$ & $-5.0 \%$ \\
\hline 100 or $120 / 120 \& 100$ & $0.1 \%$ & $0.1 \%$ & $-1.5 \%$ \\
\hline 100 or $120 / 120 \& 140$ & $3.1 \%$ & $3.0 \%$ & $0.9 \%$ \\
\hline 100 or $120 / 140 \& 80$ & $-4.4 \%$ & $-3.8 \%$ & $-5.3 \%$ \\
\hline 100 or $120 / 140 \& 100$ & $-0.5 \%$ & $-0.6 \%$ & $-1.8 \%$ \\
\hline 100 or $120 / 140 \& 120$ & $1.4 \%$ & $1.3 \%$ & $-0.3 \%$ \\
\hline
\end{tabular}

$a_{\mathrm{kVp} 1}$ was $100 \mathrm{kVp}$ for the 5-year-old and 10 -year-old phantoms and $120 \mathrm{kVp}$ for the adult phantom. 


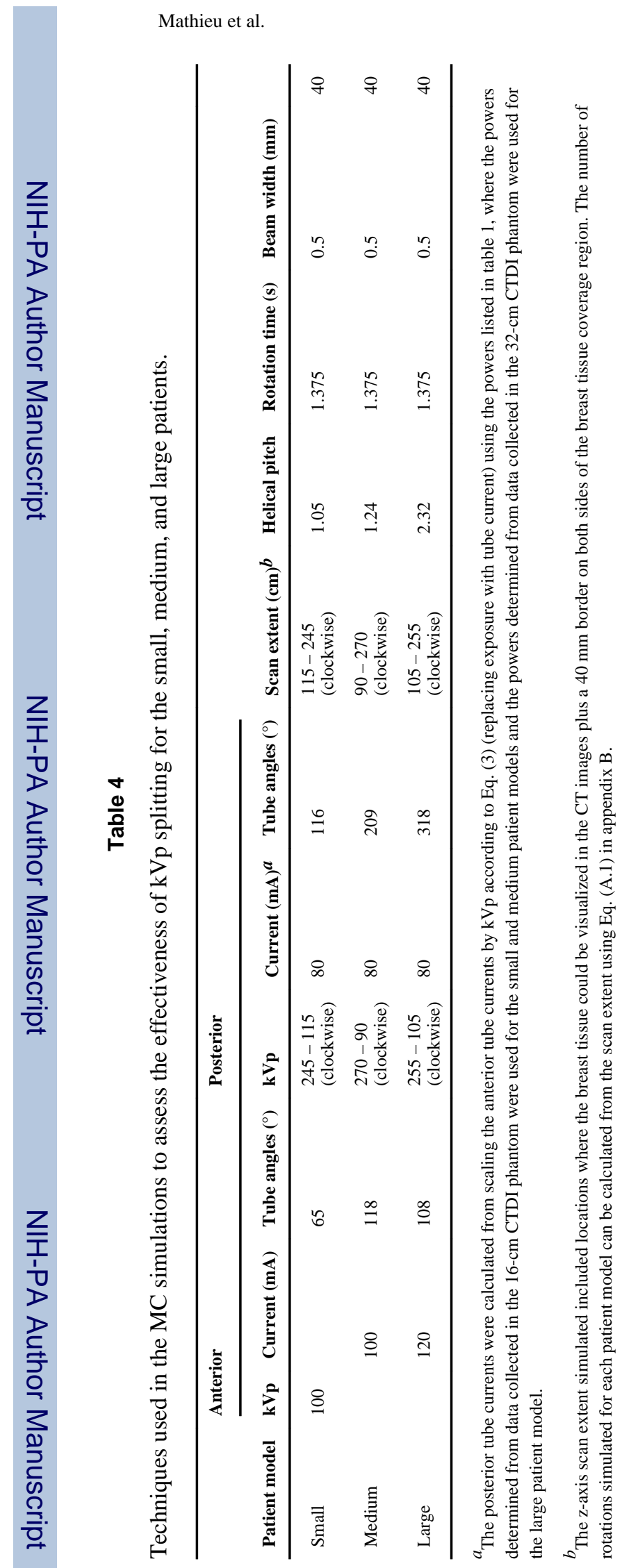

Phys Med Biol. Author manuscript; available in PMC 2014 July 07. 


\section{Table 5}

Breast and lung dose absorbed by three pediatric patients as estimated through Monte Carlo computer modeling. Dose savings (as a percentage) is given for the $\mathrm{kVp}$ splitting simulations relative to the fixed $\mathrm{kVp}$ simulation in parentheses next to the dose estimates; negative values indicate dose savings.

\begin{tabular}{llll}
\hline Patient model & $\mathbf{k V} \mathbf{p}^{\boldsymbol{a}}$ & Breast dose (mGy) & Lung dose (mGy) \\
\hline Small & 100 & 2.85 & 2.12 \\
& 100 (anterior) \& 80 (posterior) & $1.90(-33 \%)$ & $2.12(-0.1 \%)$ \\
\multirow{2}{*}{ Medium } & 100 & 4.24 & 2.48 \\
& 100 (anterior) \& 80 (posterior) & $3.82(-9.8 \%)$ & $2.35(-5.1 \%)$ \\
Large & 120 & 4.34 & 4.40 \\
& 120 (anterior) \& 80 (posterior) & $3.42(-21 \%)$ & $4.62(4.9 \%)$ \\
\hline
\end{tabular}

${ }^{a}$ The tube currents used for each $\mathrm{kVp}$ are listed in table 4. 